\title{
AGAMBEN, DELFINI E L'INDETERMINAZIONE DI VISSUTO E POETATO
}

\author{
Federico Fastelli \\ Università di Firenze
}

\begin{abstract}
Riassunto: L'intervento discute l'originale interpretazione avanzata da Giorgio Agamben a proposito dell'opera dello scrittore italiano Antonio Delfini. In particolare il saggio analizza la centralità della riflessione poetica di Delfini nel sistema estetico agambeniano: 1'intransigenza antimoderna di Delfini, infatti, è ricollegata da Agamben ad una riflessione generale sulla natura della parola poetica e relativamente alle mutazioni storiche del complesso rapporto tra opera e biografia nella cultura letteraria occidentale. In tal senso, il saggio riflette sul parallelo individuato da Agamben tra il significato profondo del racconto Il ricordo della Basca e l'idea di una "pura lingua" pre-babelica di cui parla Benjamin nel celebre intervento sul Compito del traduttore e in numerose altre occasioni. Saranno presi in esame anche altri testi dello scrittore modenese, principalmente dalla produzione in versi, tratti dalla raccolta Poesie della fine del mondo, del prima e del dopo; ma la riflessione non tralascerà anche i diari e le lettere di Delfini, cercando di offrire un ampio panorama della sua produzione.
\end{abstract}

Parole chiave: Giorgio Agamben, Antonio Delfini, Lingua e storia

Nel paradigmatico (AGAMBEN, 2008a, p. 11-34) e dichiaratamente provvisorio parterre letterario delineato dalle sue Categorie italiane, Giorgio Agamben colloca Antonio Delfini in una posizione affatto liminare. Un'estrema soglia, se vogliamo usare un linguaggio più adatto all'argomento, oppure uno "spazio d'eccezione" della propria riflessione letteraria - mutuando liberamente i termini utilizzati da Claudio Minca (MINCA, 2006, p. 387-340) che, a ben vedere, lo scrittore modenese occupa in estrema solitudine. Così, pure estendendo il discorso all'intera modernità letteraria europea, tale spazio non si affolla di ulteriori presenze, con la preziosa eccezione di Franz Kafka, che tuttavia, come ognuno sa, è già di per

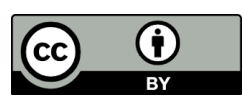

Esta obra está licenciada sob uma Creative Commons - Atribuição 4.0

\footnotetext{
${ }^{*}$ Federico Fastelli è Ricercatore di Letterature comparate all'Università di Firenze. I suoi studi si sono focalizzati soprattutto sulle avanguardie europee del secondo Novecento e sui rapporti tra letteratura e arti visive. Ha dedicato saggi all'opera di E. Sanguineti, A. Arbasino, N. Balestrini, G. Morselli, A. Robbe-Grillet, M. Butor, G. Testori, P. Bigongiari, L. Marcucci. Ha pubblicato la prima monografia sull'opera poetica di Elio Pagliarani (Dall'avanguardia all'eresia. L'opera poetica di Elio Pagliarani, Firenze, 2011). Ha scritto saggi di respiro teorico dedicati alla teoria dell'avanguardia, al teatro del Gruppo 63, alla retorica della Poesia Visiva e della Poesia Concreta, alla serialità televisiva. L'interesse per le vicende internazionali del romanzo sperimentale sono alla base della sua seconda monografia, Il Nuovo Romanzo. La narrativa d'avanguardia nella prima fase della postmodernità (1953-1973) (Firenze, 2013). Assieme a T. Spignoli, M. Corsi e M. C. Papini, ha curato il volume La poesia in immagine/L'immagine in poesia (Udine, 2014).E-mail: federico.fastelli@unifi.it;
}

Anu. Lit., Florianópolis, v. 22, n. 2, p. 25-37, 2017. ISSNe 2175-7917 
sé figura eccezionale, e si ritrova, perciò, in molti dei luoghi nevralgici della riflessione, non solo estetica, agambeniana. Insomma, se la condizione di Delfini sfiora l'unicità, è perché questi, nell'ottica del filosofo, possiede alcune qualità davvero dissuete, capaci, invero, di intercettare in uno dei suoi fuochi la propria basilare riflessione sul rapporto tra Lingua e storia (AGAMBEN, 2005). Sembra curioso, a prima vista, che questo importante compito tocchi proprio a uno scrittore che - nonostante il lavoro di alcuni autorevoli estimatori - fatica ancora oggi a confermare la propria presenza nell'asfittica costellazione della letteratura nazionale canonica. D'altra parte, considerare il paradosso semplicemente come apparente e cioè risolvere la questione in pura tautologia, dal momento che questo "stato d'eccezione" potrebbe costituire già in sé il motivo della marginalità dello scrittore, non pare ipotesi pienamente sufficiente: non lo è soprattutto per il valore di Delfini, anche considerando come l'esperienza di Kafka dimostra senza equivoco - che l'inclusione di uno scrittore in ciò che Romano Luperini definirebbe l'a parte subjecti del canone (LUPERINI apud OLIVIERI, 2001, p. 154-173), passa assai spesso dalle spigolature di posizioni certamente inconsuete o estreme o solitarie, almeno nella modernità post-baudelairiana. Né, in fondo, la ben nota professione di auto-marginalità di Delfini - ormai, del resto, ampiamente storicizzata - può essere addotta una volta di più a obbligazione infruttuosa di una fortuna memoriale così tanto avversa. Ma, il parallelo già confezionato tra la marginalità di Delfini e la sua collocazione nella categorizzazione estetica di Agamben non è sufficiente a cogliere il punto neanche in relazione al solo pensiero di quest'ultimo. Dietro 1'“ostinata distrazione della critica nei confronti dello scrittore modenese" (GUICCIARDI apud POLLICELLI, 1990, p. 195), per dirlo con Luigi Guicciardi, si deve supporre, piuttosto, l'insorgenza di frizioni ben più profonde e strutturali, come se la poetica delfiniana facesse letteralmente a pugni con una certa idea, per così dire, dominante di letteratura - e proprio in questa chiave Andrea Cortellessa ha parlato, a ragione, della "necessità" di Delfini (CORTELLESSA, 2014). Ora, anche la lettura di Agamben, se colgo il segno, si organizza proprio a partire da questo punto, e, di più, in esso trova il nucleo di tutta la capacità di quella stessa opera "di essere sviluppata" (AGAMBEN, 2008a, p. 7-8), ovverosia, con il termine utilizzato da Feuerbach e ripreso dallo stesso Agamben in Signatura rerum, la sua Entwicklungsfähigkeit.

Andando per gradi, individuiamo intanto il limes di cui qui si tratta. Scrive Agamben:

in nessuno scrittore del Novecento come in Delfini l'indeterminazione di vissuto e poetato è così assoluta e vita è veramente soltanto ciò che si genera nella parola. In questo senso, egli è l'erede più autentico della tradizione trobadorica e stilnovista $\mathrm{e}$ tutta la sua opera può essere vista come una singolare cambiale tratta a ritroso dopo sette secoli sulla cultura che ha prodotto le biografie provenzali (AGAMBEN, 2010, 
p. 78).

L’unicità di Delfini nel panorama letterario novecentesco dipende, come si capisce, dalla dialettica tra "vissuto" e "poetato": la peculiarità dello scrittore sta tutta nell'"indeterminazione assoluta" di questo rapporto, ossia, spiega Agamben, nel fatto che per Delfini "vita è veramente soltanto ciò che si genera nella parola" (AGAMBEN, 2010, p. 78). Dobbiamo fare molta attenzione a questa definizione: in primo luogo essa implica il rovesciarsi del moderno fraintendimento rispetto alla natura della parola poetica, poiché, come per i trovatori provenzali, anche per Delfini non si tratta di esprimere in parole eventi psicologici o biografici precedentemente vissuti; per i primi come per il secondo si tratta, piuttosto, del "tentativo di vivere il topos stesso, l'evento di linguaggio come fondamentale esperienza" (AGAMBEN, 2008b, p.85) biografica e artistica. Come spiega Agamben ne Il linguaggio e la morte, infatti, la svolta rappresentata dalla lirica provenzale rispetto alla retorica antica riguarda il fatto che i trovatori concepiscono l'uomo "non già sempre nel linguaggio" (AGAMBEN, 2008b, p. 84). L'ars inveniendi dell'oratore antico faceva sì ricorso ad una topica, "una tecnica dei luoghi (topoi) da cui scaturisce e inizia il discorso umano" (AGAMBEN, 2008b, p.83), ma la necessità pratica "di avere sempre a disposizione gli argomenti" (AGAMBEN, 2008b, p. 84) rese presto questa topica null'altro che una mnemotecnica, una tecnica dei luoghi di memoria, nella quale, in sostanza, il linguaggio risulta qualcosa di "sempre già dato [...], qualcosa che ha già sempre avuto luogo" (AGAMBEN, 2008b, p. 84). Per il trovatore provenzale si trattò invece di ritornare al topos originario "dell'aver luogo stesso del linguaggio" (AGAMBEN, 2008b, p. 85), non per via della memoria (in senso mnemotecnico) ma, appunto, come "esperienza dell'avvento della parola poetica" (AGAMBEN, 2008b, p. 85), a cui, "sulle tracce dell'Appetitus agostiniano" (AGAMBEN, 2008b, p. 85) i trovatori dettero il nome di Amors. L'errata idea moderna dell'amore trobadorico come sentimento privato - sempre seguendo il ragionamento agambeniano - dipenderebbe perciò dall'inesatta concezione del rapporto tra vita e poesia, e cioè, nel dettaglio, dal fraintendimento della funzione e del senso della razo nell'opera trobadorica: le razos non rappresentano, secondo Agamben, quel vissuto divenuto materia che il poeta può esprimere in poesia, ma, precisamente, ciò "che sta a fondamento della poesia e ne costituisce quello che i poeti chiamano dettato (dictamen)" (AGAMBEN, 2010, p. 76). Nel capitoletto di Idea della prosa intitolato Idea del dettato, infatti, si legge: 
sotto pena di vergogna, di saper all'occasione "aprire per prosa" (AGAMBEN, 2002, p. 33).

È proprio in questo senso, allora, che dobbiamo ripensare la dialettica tra l'opera delfiniana e quell'apparato di "vita inventata", di "vita finzionale", di cui numerosi critici si sono occupati nel tempo. Non solo l'introduzione d'autore all'edizione del 1956 dei racconti (DELFINI, 1956), su cui Agamben ritorna più volte, ma anche la premessa e le note alle Poesie della fine del mondo (DELFINI, 2013), oppure le lettere d'amore (DELFINI, 1963) ${ }^{1} \mathrm{e}$ in una certa misura (e al netto dell'arbitrio antologizzante di chi ne curò l'edizione postuma) i diari (DELFINI, 1982) dello scrittore devono essere letti come razos e messi in rapporto, quindi, con l'opera vera e propria in un senso di reciproca implicazione, piuttosto che di un movimento univocamente direzionato tra i due poli del vissuto e del poetato. È chiaro, difatti, che il tentativo di "sperimentare l'evento di linguaggio come amore" (AGAMBEN, 2008b, p. 86) dei trovatori provenzali poneva in dialettica il vissuto e il poetato; non però nel senso moderno di mettere in parole gli eventi biografici, né - ed eccoci al punto più notevole dalla questione - nel senso diametralmente opposto, per cui sarebbe immediatamente e semplicemente il poetato a fornire una giustificazione nobilitante agli eventi di un vissuto altrimenti indifendibile, fallimentare. Per Agamben, l'opera di Delfini, in questo senso, non mira soltanto, come scrive Alfredo Giuliani, a ““inventare” la vita e difendersi dalla realtà; esperimentare la vita, viverla senza schemi letterari, per poi ritrovarla nella distanza/vicinanza del ricordo, perché solo il ricordo fluttua nella duplice dimensione del vissuto e dell'inventato" (GIULIANI apud POLLICELLI, 1990, p. 82) - sebbene, come vedremo, è comunque nel segno del ricordo che dall'opera si genererà catastroficamente uno spazio d'autonomia reale del vissuto. Né la vita finzionale riproposta dalle razos è declinabile come immediata proiezione $^{2}$ di un desiderio celato o, peggio, espresso in via figurata o allegorica nell'opera (anche perché lo statuto della realtà che si rivela in queste "storie delle storie", per così dire, risulta assai poco adeguato ad una funzione di svelamento o di scioglimento dei non

\footnotetext{
${ }^{1} \mathrm{Si}$ veda quanto scrive Agamben in Categorie italiane: "consegnando poco prima di partire per il suo ultimo soggiorno romano le sue lettere d'amore a Ugo e Michin Guanda, confusi di trovarsi nelle mani un così intimo "documento d'amore", Delfini si affretta a precisare sobriamente che "si trattava dopo tutto di un'offerta editoriale"' (AGAMBEN, 2010, p. 78)

${ }^{2} \mathrm{Si}$ veda in questo senso AMORUSO, V. La vita inventata: sulla narrativa di Delfini: "così come Delfini la presenta [...] la vita inventata non è quella vissuta né quella possibile in un tempo reale, non è né il passato né il futuro ma il progetto e cioè il desiderio di essa, un vagheggiamento veramente leopardiano: come tale, tuttavia, più che un oggetto (una cosa o una persona), ha per centro se stessa, e quindi un rimando continuo, sempre vano e sempre frustrato, alla propria iterazione, è, in una parola, desiderio del desiderio, attesa dell'attendere, un movimento che contraddice la sua stessa spinta, perché non è teso in avanti, verso il fuori, ma a ritroso e dentro, nelle viscere di un tempo fuori del tempo, che su se stesso continuamente si ravvolge, e perciò, in forma e sequenza, realizza una forma di immobilità nel moto, o meglio ancora una paradossale, perché al tempo stesso incompiuta e sferica, circolarità" (AMORUSO, p. 177).
} 
detti biografici eventualmente presenti nelle opere) (CALABRESE, 2007, p. 15) ${ }^{3}$. Si tratta, invece, di comprendere il legame tra vissuto e poetato all'interno di ciò che Agamben chiama "vivere la razo" (AGAMBEN, 2010, p. 77), e di comprenderlo, s'intende, entro tutta la propria estrema indeterminazione.

Se, come abbiamo provato ad esporre, la razo «non è, dunque, né un evento biografico né un evento linguistico, ma, per così dire, una zona di indifferenza fra vissuto e poetato, un "vivere la parola" come inesauribile esperienza amorosa" (AGAMBEN, 2010, p. 76), "vivere la razo" (AGAMBEN, 2010, p. 78) significherà, propriamente, testimoniare la “teologale autenticità della scrittura" (AGAMBEN, 2010, p. 78) attraverso, per 1'appunto, il racconto finzionale del proprio "fallimento biografico" (AGAMBEN, 2010, p.78), che è espressione - appunto già in partenza destinata al fallimento - di un amore "fantasmatico", di stampo medioevale, di cui Agamben tratta diffusamente, come si sa, in Infanzia e storia:

\begin{abstract}
la scoperta medioevale dell'amore ad opera dei poeti provenzali e stilnovisti è, da questo punto di vista, la scoperta che l'amore ha per oggetto non direttamente la cosa sensibile, ma il fantasma; è, cioè, semplicemente la scoperta del carattere fantasmatico dell'amore. Ma, data la natura mediale della fantasia, ciò significa che il fantasma è, anche, il soggetto e non semplicemente l'oggetto dell'eros. In quanto, infatti, l'amore ha il suo luogo unico nella fantasia, il desiderio non trova mai davanti a sé l'oggetto nella sua corporeità $[\ldots]$ ma una immagine [...], una "nova persona" che è, letteralmente, fatta di desiderio [...], nella quale si aboliscono $i$ confini fra soggettivo e oggettivo, corporeo e incorporeo, il desiderio e il suo oggetto (AGAMBEN, 2001, p. 20).
\end{abstract}

Questo amore, si capisce, esclude radicalmente ogni sopravvivenza di psicologismo dal fatto letterario, ogni sorta di rinvenimento del sentimento in quanto stato emotivo o carattere della coscienza, e si configura precisamente come nient'altro che "intelletto d'amore", sapere, insomma, in cui "verità e bellezza comunicano" (AGAMBEN, 2015, p. 57$58)^{4}$. Si tratta dell'esperienza stessa del linguaggio, se vogliamo dirla in un altro modo, come inscindibile unità, indeterminabile, proprio, di vissuto e poetato. Antonio Delfini, in questo senso, è un poeta profondamente antimoderno: è egli, forse, il solo scrittore del Novecento ad

\footnotetext{
${ }^{3}$ In questo senso Stefano Calabrese ha parlato di "reality show". Cfr. CALABRESE, S., Antonio Delfini Verofinto. Una metalessi italiana, Udine, Forum, 2007, p. 15: «la definizione attuale di reality show - la realtà estetizzata e vissuta nella prospettiva di uno spettatore, dunque una realtà finzionale - è la più idonea a contraddistinguere il profilo antropologico di Delfini as a young man».

4 "È questo sapere, in cui verità e bellezza comunicano, che, al culmine della filosofia greca, Platone aveva fissato nella figura demonica di Eros; ed è ancora questo sapere che, alle soglie dell'età moderna, era apparso ai poeti del Duecento come "intelletto d'amore» nella figura beatificante di una Donna (Beatrice) in cui, finalmente, la scienza gode e il piacere sa. Il mitologema di Eros è necessariamente iscritto nel destino della filosofia occidentale, in quanto, al di là della scomposizione metafisica del significante e del significato, dell'apparenza e dell'essere, della divinazione e della scienza, esso fa cenno verso una salvazione integrale dei fenomeni. Sapere d'amore, filosofia, significa: la bellezza deve salvare la verità e la verità deve salvare la bellezza. In questa duplice salvazione si compie la conoscenza".
}

Anu. Lit., Florianópolis, v. 22, n. 2, p. 25-37, 2017. ISSNe 2175-7917 
affrontare esplicitamente la questione dello statuto del vissuto rispetto alla propria origine letteraria, nel segno, s'intenda, della difesa strenua e impossibile dell'autenticità della scrittura che, questo vissuto, si incarica di contenere per intero; e, d'altra parte, anche di un netto superamento - che Delfini condivide ancora con Kafka - di ogni pretesa di giustificare il reale dato biografico rendendolo autentico attraverso la scrittura ${ }^{5}$. Sommando questi due aspetti dovrebbe finalmente chiarirsi non soltanto la posizione di limite che Delfini occupa, ma più in profondità la sua sostanziale inammissibilità in ambito di canone moderno.

Per illuminare ulteriormente questo punto, comunque, dobbiamo rileggere da una specola differente la discontinuità nella poetica delfiniana costituita dalla ripubblicazione dei suoi racconti nel 1956:

Delfini, aggiungendo, nel 1956, un'introduzione alla seconda edizione dei suoi racconti, ha scritto, per Il ricordo della Basca, la più lunga razo che un poeta abbia mai immaginato per una sua opera. Ma, com'era consuetudine fra i poeti d'amore, anche in questo caso la razo può condurre il lettore fuori strada. Essa indica, infatti, immediatamente in direzione della biografia dell'autore, una biografia - s'intende inventata a partire dall'opera, ma che il lettore rischia, invece, di prendere per buona. La Basca, che è il trasparente senhal della lingua e del dettato della poesia, diventa così Isabel De Aranzadi, una ragazza conosciuta a Lerici un'estate di vent'anni prima (AGAMBEN, 2002, p. 33).

In Un enigma della Basca (AGAMBEN, 2010, p. 145-149), Agamben espone compiutamente la propria posizione sul rapporto tra il racconto in questione e la razo che lo introduce: "aprendo per prosa" il "trobar clus" del Ricordo, l'introduzione - il cui titolo originario sarebbe dovuto essere proprio Ricordo del ricordo - "mette in guardia i lettori contro la tentazione di domandare: "perché la Basca? chi è? cosa vuol dire?" (AGAMBEN, 2010, p. 145). Ora, spiega Agamben, la Basca, come ogni senhal femminile di ascendenza trobadorica e stilnovista, è ovviamente anche "simbolo della lingua della poesia" (AGAMBEN, 2010, p. 145). Si ricorderà, in effetti, come appare la Basca a Giacomo Disvetri, nel racconto:

era scesa una ragazzetta che l'aveva subito guardato sorridendo, mentre si rivolgeva al padre dicendo entonces ed altre due parole incomprensibili. "Sono spagnoli", aveva detto un ragazzo del paese. E allora Giacomo, non si sa perché, si era messo a piangere e voltando le spalle a tutti era scappato a casa (DELFINI, 1992, p. 183).

\footnotetext{
5 "La "scrittura psicologica allo specchio" che, secondo una geniale annotazione kafkiana nel penultimo quaderno in ottavo, ci fa sembrare che gli uomini siano incessantemente occupati a consolidare la propria vita con scritture e giustificazioni a posteriori, è qui raddrizzata con un gesto deciso a mostrare, contro ogni lettura psicologica, che "in realtà l'uomo erige la sua vita sulle proprie giustificazioni", poiché "nessuno qui crea altro che la sua possibilità di vita spirituale". È su questi archetipi che, in ogni caso, tanto Delfini che Kafka hanno costruito la loro vita. Il loro fallimento biografico (o, almeno, quello che ci appare tale nella scrittura rovesciata della psicologia) doveva testimoniare della teologale autenticità della scrittura (del suo porsi in $\operatorname{arché}$ ), e non questa giustificarlo". (AGAMBEN, 2002, p. 76)
}

Anu. Lit., Florianópolis, v. 22, n. 2, p. 25-37, 2017. ISSNe 2175-7917 
Ed ecco invece come il racconto si scioglie nella razo:

un signore dai lineamenti fini e dal portamento oltremodo signorile, molto dimesso negli abiti per quanto fossero eleganti, stava pensoso, tenendosi un po' in disparte dai suoi figlioletti (un bambino e una ragazzetta): i quali stavano conversando in una lingua di tale toccante dolcezza, che il mio cuore a udirli, sembrò volesse fermare il proprio battito per lasciare le cose sospese per sempre in quell'attimo (AGAMBEN, 1956, p. 85).

Il "perché" il "chi è" e "il che vuol dire" la Basca costituiscono interrogazioni invalicabili dell'esperienza artistica delfiniana: l'autenticità dell'esperienza linguistica della poesia attestata attraverso la razo sta tutta, se ben si guarda, nel fatto che la Basca, appunto, è una donna che, pure, è una lingua ignota, appunto la lingua basca; ovvero, per reciproco, una lingua che, pure, è una donna amata e sconosciuta. Con ogni evidenza ciò trascende qualsiasi significato realmente biografico, e, per quanto abbiamo detto, si configura come esperienza medesima della lingua poetica, o, più propriamente, come esperienza attraverso la lingua poetica del fantasma quale soggetto-oggetto di amors. È infatti alla fantasia che occorre guardare per comprendere lo statuto angelico - in senso stilnovistico - della Basca, che si presenta al ricordo del protagonista del racconto, come i lettori di Delfini ricorderanno, proprio come "immaginazione pura e separata dal corpo" (AGAMBEN, 2001, p. 20):

Gli era nata un'immagine: qualcosa come una fanciulla. Aveva sentito dentro di sé il
suono di una voce lenta e brillante al tempo stesso, chiara, serena, resa emotiva da
certe inflessioni, impossibili a descrivere, come potrebbero forse essere i raggi della
luna illuminati su un prato su cui un gatto si rotola modulando suoni di
soddisfazione e tramandando dagli occhi le segnalazioni di un faro (DELFINI, 1956,
p. 180).

Solo più tardi, effettivamente, "dalla voce si venivano via via formando delle sembianze" (DELFINI, 1956, p. 181). Ed è anche interessante notare, per inciso, che il narratore parli esplicitamente di "risveglio", intendendo riappropriazione della facoltà dell'esperienza (dell'amore, del linguaggio), contrapposta alla conoscenza, al pensiero («si era accorto di non pensare più»), e il riferimento, se non ci si inganna, è precisamente all'espropriazione moderna del quotidiano come materia prima dell'esperienza:

a Giacomo il risveglio era venuto improvvisamente, come suole accadere nella maggior parte di questi casi. Era stato quest'anno, una sera che usciva prima del solito dall'ufficio: fu un attimo. Si era accorto di non pensare più alla banca, ai discorsi fatti al caffè, alle solite sconcezze che fanno ridere con una specie di meccanica malata della bocca, ai luoghi comuni, ai pregiudizi, alle cose dei giornali, agli avvenimenti tutti della vita corrente, al risonare dei grandi e piccoli motivi musicali, al cinema, ecc. (DELFINI, 1992, p. 180-181)

Che, poi, è esattamente quanto Agamben descrive del primo paragrafo di Infanzia e 
storia: "l'uomo moderno torna a casa la sera sfinito da una farragine di eventi - divertenti o noiosi, insoliti o comuni, atroci o piacevoli - nessuno dei quali è però diventato esperienza" (AGAMBEN, 2001, p. 6). In ogni caso, tornando alla questione della Basca come senhal trobadorico e stilnovistico, la riflessione del filosofo si sofferma sulla composizione poetica apparentemente glossolalica che compare a conclusione del racconto, e che risulta, in verità, scritta proprio in basco (AGAMBEN, 2010, p. 147-148). L'impressione glossolalica, infatti, sorregge per intero il compito della poesia, ossia indica il suo statuto di esperienza della parola come impossibile ricordo di una lingua "in cui lo spirito si confonde immediatamente con la voce" (AGAMBEN, 2010, p. 146), di qualcosa cioè - la Basca - "che è talmente intimo e presente da non poter essere in nessun caso ricordato" (AGAMBEN, 2010, p. 146): insomma è proprio il ritornare della lingua al momento topico dell'aver luogo del linguaggio ciò di cui qui si parla, ed equivale - in fondo - alla ferma consapevolezza che lo stesso linguaggio non è sempre già dato; o ancora, per dirla in altri termini, che l'uomo ha, precisamente, secondo la terminologia agambeniana, un'infanzia:

\begin{abstract}
se non ci fosse l'infanzia dell'uomo, certamente la lingua sarebbe un "gioco", la cui verità coinciderebbe con il suo uso corretto secondo regole logico-grammaticali. Ma, dal momento che vi è un'esperienza, che vi è un'infanzia dell'uomo, la cui espropriazione è il soggetto del linguaggio, il linguaggio si pone allora come il luogo in cui ogni esperienza deve diventare verità (AGAMBEN, 2001, p. 49).
\end{abstract}

Come si capisce da quest'ultimo passaggio, la rilevanza che Agamben attribuisce a Delfini, l'estrema soglia in cui lo convoca, l'insistenza con cui più volte ritorna sugli stessi argomenti dipendono dal fatto che con Il ricordo della Basca prima, con Le poesie della fine del mondo poi, e, in generale, con un atteggiamento poetico costantemente volto verso il "mito di una lingua in cui lo spirito si confonde - almeno in apparenza - immediatamente con la voce" (AGAMBEN, 2002, p. 33), lo scrittore modenese chiama in causa "lo statuto assolutamente primordiale del linguaggio" (AGAMBEN, 2010, p. 74), la "dimora del logos nell'arche”" (AGAMBEN, 2010, p. 74), ovverosia la negatività fondamentale che, da sempre, segna e contraddistingue l'opera poetica, e di cui la Basca costituisce l'enigma, e, ad un tempo, il postremo vessillo ${ }^{6}$. Ebbene la Basca delfiniana rappresenta esattamente "ciò che resta indicibile e non detto in ogni lingua" (Agamben, 2005, p. 44) e che infine coincide "proprio [con] ciò che essa intende e vuol dire: la pura lingua, la parola inespressiva" (Agamben, 2005, p. 44). Questa lingua così sconosciuta eppure così amata allude, nei termini

\footnotetext{
${ }^{6}$ Agamben affronterà questi argomenti anche in La comunità che viene (Torino: Einaudi, 1990), Mezzi senza fine. Note sulla politica (Torino: Bollati e Boringhieri, 1996) e L'aperto. L'uomo e l'animale (Torino: Bollati e Boringhieri, 2002).
}

Anu. Lit., Florianópolis, v. 22, n. 2, p. 25-37, 2017. ISSNe 2175-7917 
benvenistiani ripresi da Agamben in Infanzia e storia, al puro ordine semiotico di ogni lingua, in contrapposizione all'ordine semantico ${ }^{7}$. Allude insomma a ciò che è proprio del segno linguistico prima che esso si faccia discorso, oppure, per un altro verso, a ciò che non può essere trasposto da una lingua ad un'altra, per esempio nella pratica della traduzione ${ }^{8}$. È la lingua prima della storia, la pura lingua dell'infanzia dell'uomo e in quanto tale essa non deve essere compresa, ma, semplicemente riconosciuta:

Il semiotico (il segno) deve essere RICONOSCIUTO; il semantico (il discorso) deve essere COMPRESO. La differenza tra riconoscere e comprendere rinvia a due facoltà distinte dello spirito: quella di percepire l'identità fra anteriore e attuale, da una parte, e quella di percepire il significato di una enunciazione nuova, dall'altro (AGAMBEN, 2002, p. 53).

"Perché, la Basca?", "chi è la Basca?", "che cosa vuol dire la Basca?" sono, lo ripetiamo, domande impossibili. È forse proprio in questo senso che l'opera di Delfini è ricordata da Agamben anche in chiusura dell'intervento Lingua e storia. Categorie linguistiche e categorie storiche in Walter Benjamin, come coraggioso (e raro) esperimento di pensare - benjaminianamente, proprio - "una comunità umana e una lingua umana che non rimandassero più ad alcun fondamento indicibile $\mathrm{e}$ non si destinassero più $\mathrm{a}$ un tramandamento infinito, e in cui le parole non si distinguessero più da ogni altra prassi umana" (AGAMBEN, 2005, p. 54). In altre parole, Il ricordo della Basca si può leggere, se si coglie correttamente lo spunto, come l'impossibile tentativo, già pregiudicato in principio, e proprio perciò di impareggiabile coraggio, di scavalcare la "condizione decaduta del linguaggio, sancita dalla confusione babelica delle lingue" (AGAMBEN, 2005, p. 42) - dalle lingue nella storia - di cui Benjamin parla nel celebre intervento sul Compito del traduttore, e in diverse altre occasioni, per giungere (e/o tornare), insomma, a quella "pura lingua" dell'"umanità redenta", ovvero a quella "parola che non vuol più dire, che non si destina più al tramandamento storico di un significato" (AGAMBEN, 2005, p. 43).

Eppure, come ho già accennato, la composizione poetica che chiude il racconto delfiniano è solo in apparenza una pura glossolalia e ciò spiega non soltanto il significato profondo del titolo Ricordo della Basca, ma la straordinaria consapevolezza dell'operazione estetica di Delfini. Come precisa lo stesso Agamben:

\footnotetext{
7 "Il semiotico designa il modo di significazione che è proprio del SEGNO linguistico e che lo costituisce come unità [...]. Col semantico entriamo nel modo specifico di significazione generato dal DISCORSO" (BENVENISTE apud AGAMBEN, 2001, p. 53).

8 "Si può trasporre il semantismo di una lingua in quello di un'altra "salva veritate": è la possibilità della traduzione; ma non si può trasporre il semiotismo di una lingua in quello di un'altra: è l'impossibilità della traduzione. Si tocca qui la differenza del semiotico e del semantico" (BENVENISTE apud AGAMBEN, 2001, p. $54)$.
} 
Il racconto s'intitola, tuttavia, Ricordo della Basca, a significare che la scrittura è il tentativo, condannato in partenza, di afferrare proprio quest'immemorabile prossimità, quest'amore inallontanabile (di qui 'l'irrimediabile tragedia di questo ricordo"). Del resto, la poesia, di cui il racconto stesso è la razo, non è, in realtà, una glossolalia, ma una copla in purissima lingua basca, che, tradotta, si conclude con questi versi: "quando trovo la poesia / tu ti stai addormentando; / come il sogno della notte / sia per te il mio canto" (AGAMBEN, 2002, p. 33-34).

Che significa, quindi, in termini più generali, che:

l'esperienza della lingua poetica (cioè dell'amore) è interamente compresa nella scissione fra una presenza immemorabile e un poter soltanto ricordare. La lingua della poesia non è, dunque, una perfetta glossolalia, in cui la scissione si sutura, così come nessuna lingua umana, malgrado la sua tensione verso l'assoluto, può mai, scavalcando la mediazione del senso, risolversi senza residui in un "parlare in lingua" (AGAMBEN, 2010, p. 147).

Il fallimento di questo approdo è proprio l'impossibilità del ricordo della Basca: "la scomparsa della Basca è eterna, perché essa manca eternamente nella lingua degli uomini, in cui si attesta solo attraverso il babelico discordo dei molteplici idiomi" (AGAMBEN, 2010, p. 147). Il fallimento del ricordo che è al centro del racconto delfiniano, e la razo, che costituisce il ricordo di questo "fallimento del ricordo", ovvero lo scioglimento del suo dettato - in prosa, appunto, secondo i termini agambeniani - certificano quindi una scissione intrinseca al linguaggio dell'uomo tra lingua e discorso e preparano, evidentemente, l'estremo e doloroso approdo della carriera di Delfini alle Poesie della fine del mondo. Se infatti il dettato poetico che struttura Il ricordo si regge, per forza di fede, sul fantasmatico amore per la Basca, e il linguaggio resta quindi il luogo dove l'esperienza si fa verità, a differenza della biografia reale - "a ripensarci dico che se avessi allora tenuto un journal non avrei potuto avere il tempo di vivere, né l'estro di creare, quei veri racconti, vivendo i quali non ho avuto il tempo di scriverli” (DELFINI, 1956, p. 11) - le Poesie della fine del mondo, come Agamben rileva, indicano una "lacerazione, che abbandona la vita alla sua "vera mala sorte"" (AGAMBEN, 2010, p. 79) e che "si riverbera, però, immediatamente sulla poesia stessa, divenuta ora "mala poesia", che, tuttavia, il poeta non può non scrivere [...] Il poeta stesso deve, cioè - ed è questo, nella catastrofe, l'evento più atroce -, rompere il proprio dettato" (AGAMBEN, 2010, p. 79). E in questo ultimo senso, davvero, la lettura agambeniana sembra cogliere nel segno e penetrare in profondità il testo dello scrittore. Convince, in particolare, che proprio da tale rottura dipenda "l'inversione della figura femminile, cui i poeti d'amore consegnavano l'immagine più integra del loro dettato" (AGAMBEN, 2010, p. 80), che organizza l'intera raccolta, e su cui la critica ha creduto sovente di poter specchiare brutalmente il riflesso di noti dati biografici. Si rilegga la Premessa alle poesie: 
Il poeta ricorda, si rimorde, vede e intravede. "È tutta colpa di quella data storia", dichiara. Poi si ricrede. Il poeta non è più un poeta: è un assassino, un ucciso. C'è una signora che l'osserva. "È lei la causa della fine del mondo. È una donna, che ha fatto perire ogni cosa!". Quella signora che lo sta osservando mentre muore è il simbolo della frode, del tradimento e del peccato (DELFINI, 2013, p. 97).

E si veda la coerenza della lettura agambeniana:

la donna (la Basca, iscritta nella tradizione dei senhal stilnovisti e provenzali, fra Beatrice, Giovanna, Miellz de Domna, Dezirada, Bon Vezi), che portava la cifra dell'unità di poetato e vissuto, della vita nella lingua, dischiavata ora a forza dalla penna e dalla parola, si rovescia in nuda vita, simbolo orrido e oscuro "della frode, del tradimento, del peccato" (AGAMBEN, 2010, p. 80).

E si rileggano infine, da questa prospettiva - e quindi dall'inesorabile condizione di questo poeta divenuto assassino adesso, e per sempre "condannato a uccidere la sua "signora", cioè la sua stessa vita e la sua stessa poesia, la sua vita-poesia" (AGAMBEN, 2010, p. 79) testi come Non più una storia, la vita, o Fin tanto, o ancora A Cesena, L'unica via possibile è la morte, Sono stanco, solo per citare i più rivelatori, in tal senso. Si pensi, per esempio, a versi come

Per levarmi i fantasmi d'attorno un medico di Roma mi ha ordinato i calmanti: non più sarà discorso per te d'amore e di amanti ma solo di avvocati merdosi e di soldi contanti. (DELFINI, 2013, p. 124)

nei quali, esplicitamente, Delfini mette in gioco la distruzione di quel concetto "fantasmatico" di Amore che dava accesso ad una soddisfazione sempre mediale e sempre esaudita, e che non è altro che la poesia stessa, una poesia vuota, che mette in scena il linguaggio in quanto esperienza autentica, originaria e assolutamente infondata. Quella poesia è la vita autentica: l'unica nella quale l'amore sa riunire in sé il desiderio - "legato alla fantasia insaziabile e incommensurabile" (AGAMBEN, 2001, p. 21) - e il bisogno - "legato alla realtà corporea, misurabile e teoricamente soddisfacibile" (AGAMBEN, 2001, p. 21). Ma quando lo spazio della fantasia entra, implacabilmente, anche per Delfini, nell'ordine dell'irreale - "per levarmi i fantasmi d'attorno / il medico mi ha ordinato i calmanti" (DELFINI, 2013, p. 124) - lì si spezza, inesorabile, l'incanto di questa lingua che precede il discorso e ogni significato: l'amore si scinde ora nelle sue due componenti e il desiderio diventa insoddisfacibile. Qui non vi è spazio che per la mala poesia: niente più "discorso per te d'amore e di amanti" (DELFINI, 2013, p. 124) ma soltanto la villania della reale sfera biografica, o, per chiudere in mala-poesia, quel mondo "di avvocati merdosi e di soldi contanti” (DELFINI, 2013, p. 124). 


\section{Bibliografia}

AGAMBEN, Giorgio. Infanzia e storia: distruzione dell'esperienza e origine della storia. Torino: Einaudi, 2001.

. Idea del dettato. In: . Idea della prosa. Macerata: Quodlibet, 2002.

. La potenza del pensiero: saggi e conferenze. Vicenza: Neri Pozza, 2005.

. Signatura rerum: sul metodo. Torino: Bollati e Boringhieri, 2008a.

$2008 b$

. Il linguaggio e la morte: un seminario sul luogo della negatività. Torino: Einaudi,

Categorie italiane: studi di poetica e di letteratura. Roma-Bari: Laterza, 2010.

Gusto. Macerata: Quodlibet, 2015.

CALABRESE, Stefano. Antonio Delfini Verofinto: una metalessi italiana. Udine: Forum, 2007.

CORTELLESSA, Andrea. Antonio Delfini: l'attacchino metafisico. In: Alfabeta 2. Disponibile su: www.alfabeta2.it/2014/02/16/antonio-delfini-lattacchino-metafisico. Ultimo accesso: 10/09/2017.

DELFINI, Antonio. Il ricordo della Basca. Dieci racconti e una storia. Pisa: Nistri-Lischi, 1956.

Lettere d'amore e Ritorno in città. Parma: Guanda, 1963.

. Il ricordo della Basca. Milano: Garzanti, 1992.

. Poesie della fine del mondo, del prima e del dopo. Torino: Einaudi, 2013.

DELFINI, Antonio. GUINZBURG, Natália. (Org.) Diari 1927-1961. Turim: Einaudi, 1982.

GIULIANI, Alfredo. Le "poesia della fine del mondo". In: POLLICELLI, C. et al. (Org.). Antonio Delfini: Testimonianze e saggi. Modena: Mucchi, 1990.

GUICCIARDI, Luigi. Cinquant'anni di disagio: la critica e Delfini. In: POLLICELLI, Cinzia et al. (Org.). Antonio Delfini: testimonianze e saggi. Modena: Mucchi, 1990.

LUPERINI, Romano. La questione del canone, la scuola e lo studio del Novecento. In: OLIVIERI, Ugo. (Org.). Un canone per il terzo millennio. Testi e problemi per lo studio del Novecento tra teoria della letteratura, antropologia e storia. Milano: Mondadori, 2001.

MINCA, Claudio. Giorgio Agamben end the new biopolitical Nomos. In: In: Geografiska Annaler. Series B, Human Geography, V. 88, n. 4, 2006, p. 387. 


\section{Agamben, Delfini e a ideterminação de vivido e poetado}

Resumo: $O$ ensaio discute a interpretação original de Giorgio Agamben sobre a obra do escritor italiano Antonio Delfini. Em particular, o ensaio analisa a centralidade da reflexão poética de Delfini no sistema estético agambeniano: a intransigência antimoderna de Delfini é, de fato, ligada por Agamben a uma reflexão geral sobre a natureza da palavra poética e relativa às mutações históricas da complexa relação entre obra e biografia na cultura literária ocidental. Nesse sentido, o ensaio reflete sobre o paralelo identificado por Agamben entre o significado profundo do conto Il ricordo della Basca e a ideia de uma "pura língua" prébabelica falada por Benjamin em seu famoso trabalho na Tarefa do tradutor e em muitas outras ocasiões. Serão examinados também outros textos do escritor de Môdena, principalmente da produção em versos, escolhidos na coletânea Poesie della fine del mondo, del prima e del dopo; mas a reflexão não deixará de lado também os diários e as cartas de Delfini, buscando oferecer um panorama amplo de sua produção.

Palavras-chave: Giorgio Agamben. Antonio Delfini. Língua e história.

Recebido em: 27/10/2017 Aceito em: 09/11/2017

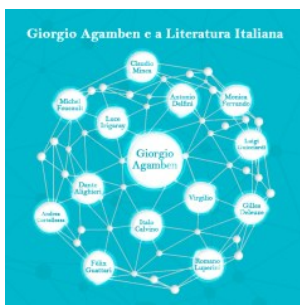

